\title{
Saga of Superfluid Solids
}

\author{
Vyacheslav I. Yukalov ${ }^{1,2}$ \\ 1 Bogolubov Laboratory of Theoretical Physics, Joint Institute for Nuclear Research, Dubna 141980, Russia; \\ yukalovtheor.jinr.ru \\ 2 Instituto de Fisica de São Carlos, Universidade de São Paulo, CP 369, São Carlos, São Paulo 13560-970, Brazil \\ Received: 12 November 2019; Accepted: 3 February 2020; Published: 7 February 2020

Abstract: The article presents the state of the art and reviews the literature on the long-standing problem of the possibility for a sample to be at the same time solid and superfluid. Theoretical models, numerical simulations, and experimental results are discussed.

Keywords: solid state; superfluid state; diagonal order; off-diagonal order; translational symmetry; global gauge symmetry

\section{Introduction}

The term "superfluidity" was coined by Kapitza, who actually discovered the phenomenon of superfluidity in liquid Helium, in his paper [1] followed by the paper by Allen and Misener [2]. Kapitza had set up an experiment with two cylinders that were connected by a thin tube. Only below the $\lambda$ point, helium was flowing easily through the tube, suggesting a strikingly low viscosity. The first explanation of superfluidity, in the frame of the two-fluid model, was suggested by Landau [3-5] and Tisza [6]. The key evidence establishing the two-fluid model of superfluidity was given by Andronikashvili [7-9] studying the period and damping of torsional oscillations of stacked closely spaced rotating disks. More details about the interesting history concerning the discovery of and first works on superfluidity can be found in Refs. [10-13].

However, the subject of this review is not the description of superfluidity in liquids, but a rather unexpected problem considering the possibility of superfluid effects in solids. This review gives a brief account on the history and the present-day situation on the possibility for a solid to be at the same time superfluid. This puzzle has a long history that at the present time is intensively explored. Historically, the assumption that a solid could display superfluid properties was considered for solid helium, and most works have been devoted to this material. Recently, the simultaneous breaking of translational symmetry, typical of crystals, and global gauge symmetry, usually accompanying superfluids, has been displayed for trapped dipolar gases under special conditions (Bose-Einstein condensation, tuned atomic interactions, and aligned magnetic dipoles).

The review attempts to present the overall portrait, touching only the basic ideas, sufficient for grasping the outline of the whole picture, without much plunging into specific details. The brevity of the narration is compensated by a quite comprehensive list of literature. Some more information on concrete details from the works before 2012 can be found in the review articles [14-16].

The main aim of the present review is to suggest a general description that, although being precisely formulated, would be understandable for wide audience, where not all are professionals in the field of quantum condensed matter.

\section{What Is a Superfluid Solid}

To the first glance, the topic announced in the title sounds as an oxymoron. The common wisdom teaches us that a solid is rigid, keeping a fixed shape, while a superfluid is a substance with no fixed 
shape and no viscosity, easily flowing without friction. Thus, in the Merriam-Webster dictionary a solid is defined as "a substance that does not flow perceptibly under moderate stress, has a definite capacity for resisting forces (such as compression or tension) which tend to deform it, and under ordinary conditions retains a definite size and shape". In the same dictionary a superfluid is classified as "an unusual state of matter...characterized by apparently frictionless flow".

Of course, the ability to flow without friction is not the sole characteristic of a superfluid which possesses other exotic properties. Thus, an important feature of a superfluid is the appearance of quantized vortices under rotation. A quantum vortex differs from the familiar classical vortices in turbulent liquids, such as water, by the quantization of circulation, when the integral of velocity around the core of a vortex is proportional to $(2 \pi \hbar / m) n$, where $\hbar$ is the reduced Planck conatnt, $m$ is the atomic mass, and $n$ is an integer. The other remarkable property of a superfluid is the fountain effect that causes liquid helium, being heated, to flow up the sides of open containers.

Regarding friction, it is useful to mention that there exist classical liquids composed of self-propelled particles that can organize themselves to counterbalance the energy loss resulting from viscous dissipation and thereby dramatically lower the fluid's viscosity, driving it to vanish or even to become negative [17]. However, a frictionless flow with no energy dissipation is usually considered to be a hallmark of superfluidity (and superconductivity).

It looks that such contradictory terms as solid and superfluid could not be met in conjunction. Nevertheless, the idea that solids could have some superfluid properties has been advanced in theoretical works and has been intensively studied both theoretically and experimentally till presently.

To correctly understand the subject, it is necessary, first, to recollect the definitions of what is solid state and what is superfluid state.

In the common parlance, solid state is a rigid state of matter, being able to keep its shape for very long time, provided that external forces do not overpass threshold values. Such external forces, which could destroy a solid sample, could be pressure, temperature, or chemical impact. Otherwise, when these external forces are below their thresholds, a solid sample can survive for very long time.

Usually, solids are arranged in crystalline structures. Such spatially periodic or quasiperiodic structures are said to possess diagonal long-range order. One also says that in crystals translational uniform symmetry is spontaneously broken, as compared with translationally uniform liquids. It is important that the symmetry breaking is spontaneous, i.e., self-organized. Thus, if one imposes an external periodic field upon liquid or gas, they acquire some periodic properties. However, anyway, they are liquid or gas, not solids.

Periodic structures can sometimes self-organize in nonequilibrium liquid or gaseous systems for a short period of time. Clearly, such temporally periodic systems have nothing to do with solids, even if their periodicity has appeared spontaneously. For example, quantum Bose gases can be Bose-condensed, with exhibiting superfluid properties. Upon their interactions, during some nonequilibrium processes, they can form temporal interference patterns. In that sense the system demonstrates spontaneous breaking of uniform translational symmetry. Such gases can be temporarily superfluid and periodically arranged in an interference pattern. However, they are not solids, they are gases. In addition, they are nonequilibrium, exhibiting interference patterns for a finite, usually very short, time.

There exist as well the terms "amorphous solids" or "glasses" that do not exhibit long-range periodic spatial order. They may look like genuine solids, being often rather rigid and being able to keep their shape for long time. However, these materials are not absolutely stable, but metastable, and tend to crystallize with time, although this time can be very long, sometimes tens or hundreds of years.

The intermediate case between the crystals, with ideally periodic lattices, and amorphous solids, with random locations of their constituents, are the crystals with defects. Crystals with defects, such as vacancies, interstitials, and dislocations, are mainly periodic, but the strict periodicity is absent around defects. Actually the majority of real crystals contain some defects, except maybe rare especially prepared samples. 
Summarizing, it is possible to accept the following convention: Solid state is a rigid state of matter that in equilibrium is stable or metastable in a finite region of parameters, such as pressure and temperature, or some external fields, where it is able to keep its shape for long time.

Contrary to solids, superfluids are fluids that cannot keep a rigid shape, but can flow without friction and exhibit such features as quantized vortices and fountain effect. The widely known examples are ${ }^{3} \mathrm{He}$ and ${ }^{4} \mathrm{He}$.

A solid that would combine the features of rigidity and superfluidity would deserve the name of superfluid solid. In the literature, one often calls such superfluid solids as "supersolids", as suggested by Matsuda and Tsuneto [18] and Mullin [19]. This name, however, looks a bit confusing. The standard meaning of the word "super" accentuates the given property, but does not contradict it. For instance, "superradiance" means superstrong radiance. "Superconductivity" implies superstrong conductivity. "Superfluidity" signifies superstrong fluidity. Then "supersolidity" should assume superrigid solidity. However, vice versa, one talks about a solid with some superfluid properties. Also, the term "supersolid" has already been used for many years with respect to crystals in space dimensionality larger than three [20].

One often names a "supersolid" any system, where simultaneously there occurs uniform translational symmetry breaking, typical of solids, and global gauge symmetry breaking, typical of superfluids. In that sense, interfering Bose-condensed gases should be termed "supersolids", which sounds a bit strange. Interfering gases are anyway gases, even if they are spatially periodic. In addition, superfluidity does not necessarily require global gauge symmetry breaking.

Thus, a superfluid solid can be classified as such, provided it enjoys the following mutually complimenting properties.

(i) It must be a solid that is a rigid system being able to keep for long time its shape in a finite range of external conditions. Long time implies the time that is much longer than any of the characteristic internal times, such as interaction time and local equilibration time.

(ii) It must possess the above properties being in an equilibrium state, meaning either an absolutely equilibrium state or a metastable state. This does not impose the requirement of necessarily being periodic. Amorphous solids also are solids. In addition, crystals with defects are not ideally periodic.

(iii) It must demonstrate, inside its volume, superfluidity as a frictionless motion of matter. This may be connected with the spontaneous breaking of gauge symmetry, at least locally, although this is not compulsory.

These properties are kept in mind throughout the review when we talk about superfluid solids. To stress it once more, the latter are not necessarily ideally periodic, but have to be rigid and stable. Interfering periodically modulated Bose gases are interesting objects that, however, are not solids. In addition, they are usually unstable. Superfluidity occurring in external periodic potentials, for instance in optical lattices, also does not constitute superfluid solids, since such externally imposed lattices are not self-organized.

\section{Model of Coherent Crystal}

A Bose system at zero temperature and weak interactions can be almost completely Bose condensed, which means that the system is in a coherent state. Then such a system can be described in the quasi-classical approximation. The standard Hamiltonian in this approximation reads as

$$
\begin{gathered}
H=\int \varphi^{*}(\mathbf{r}, t)\left(-\frac{\nabla^{2}}{2 m}+U\right) \varphi(\mathbf{r}, t) d \mathbf{r}+ \\
+\frac{1}{2} \int \varphi^{*}(\mathbf{r}, t) \varphi^{*}\left(\mathbf{r}^{\prime}, t\right) \Phi\left(\mathbf{r}-\mathbf{r}^{\prime}\right) \varphi\left(\mathbf{r}^{\prime}, t\right) \varphi(\mathbf{r}, t) d \mathbf{r} d \mathbf{r}^{\prime},
\end{gathered}
$$


where $\varphi(\mathbf{r}, t)$ is a complex-valued function (not operator) of the coordinate vector $\mathbf{r}$ and time $t$ called condensate function, $U=U(\mathbf{r}, t)$ is an external potential, and $\Phi(\mathbf{r})$ is an interaction potential. The Planck constant is set to one. The equation of motion

$$
i \frac{\partial}{\partial t} \varphi(\mathbf{r}, t)=\frac{\delta H}{\delta \varphi^{*}(\mathbf{r}, t)}
$$

yields the Nonlinear Schrödinger equation

$$
i \frac{\partial}{\partial t} \varphi(\mathbf{r}, t)=\left(-\frac{\nabla^{2}}{2 m}+U\right) \varphi(\mathbf{r}, t)+\int \Phi\left(\mathbf{r}-\mathbf{r}^{\prime}\right)\left|\varphi\left(\mathbf{r}^{\prime}, t\right)\right|^{2} d \mathbf{r}^{\prime} \varphi(\mathbf{r}, t) .
$$

Equation (3) was introduced by Bogolubov in his well-known book Lectures on Quantum Statistics [21], which has been republished many times, for instance, in [22-24]. Gross [25-31] has intensively studied this equation showing that it possesses nonuniform solutions, including stationary periodic solutions [26-29], when particle interactions contain an attractive part. Since the coherent condensate function corresponds to the Bose-Einstein condensate that supports superfluidity, the periodic Gross solution describes a periodic superfluid system. This could be treated as the first model of superfluid solid, provided it would be proved that it is really related to a solid, but not just to a periodic gas. However, the found periodic solution looks to be more appropriate to a periodically modulated superfluid than to a solid [26-28].

Similar idea of coherent crystallization was considered later for more refined models [32,33]. The essential point in such models is the requirement that particles, being in the same coherent state, could freely move either over the whole volume of the system, or at least over some of its macroscopic parts. Without such a motion, the system cannot be coherent and Bose-Einstein condensation cannot occur [34].

\section{Model of Vacancion Superfluidity}

Andreev and Lifshitz [35] suggested that superfluidity can arise in quantum crystals with defects. Since vacancies are essentially more mobile than impurities [36], they can form a kind of a quantum liquid of vacancions inside the crystal and superfluidity can develop [37,38].

Chester $[39,40]$ argued that Bose-Einstein condensation with zero momentum (hence superfluidity) should appear in any quantum crystal described by the ground-state many-body wave function of the Jastrow type

$$
\Psi\left(\mathbf{r}_{1}, \mathbf{r}_{2}, \ldots\right) \propto \prod_{i \neq j} f\left(r_{i j}\right),
$$

in which $r_{i j} \equiv\left|\mathbf{r}_{i}-\mathbf{r}_{j}\right|$ and

$$
f(r)=\exp \left\{-\frac{1}{2} u(r)-\chi(r)\right\},
$$

where $u(r)$ is real, bounded from below and has a finite range, which is to say

$$
u(r) \propto \frac{\text { const }}{r^{3+\varepsilon}} \quad(r \rightarrow \infty),
$$

with $\varepsilon>0$, while $\chi(r)$ is finite and, either is zero or, for large $r$, behaves as

$$
\chi(r) \propto \frac{\text { const }}{r^{2}} \quad(r \rightarrow \infty) .
$$

However, in an ideal crystal, according to Penrose [34], Bose-Einstein condensation cannot arise.

Several experimental attempts have been undertaken trying to discover superfluidity in solid ${ }^{4} \mathrm{He}$. An overview of these unsuccessful searches, accomplished till 1992, is given by Meisel [41]. 


\section{Nonclassical Rotation Inertia}

Leggett [42] mentioned that the tentative appearance of superfluid fraction in a solid could be found by measuring the moment of inertia $I(T)$ of the studied system as a function of temperature, as has been suggested earlier for liquids by Andronikashvili [7-9]. Thus, the rotational moment of inertia can be connected with the superfluid fraction by the relation

$$
I(t)=I_{0}\left[1-\frac{\rho_{s}(T)}{\rho}\right],
$$

where $I_{0}(T)$ is the classical moment of inertia for the whole system and $\rho$ is average density. Upon the appearance of superfluid density $\rho_{s}(T)$, the rotational moment of inertia decreases and, respectively, the oscillation frequency of the sample, proportional to $1 / \sqrt{I(T)}$, increases, while the resonant oscillation period $T_{o s c}=2 \pi / \omega \propto \sqrt{I(T)}$ decreases.

Kim and Chan [43-46] accomplished a series of experiments with a torsional oscillator technique for ${ }^{4} \mathrm{He}$, claiming the discovery of superfluidity at low temperatures below about $200 \mathrm{mK}$. These measurements were followed by many other experiments announcing their agreement with the Kim-Chan results [47-56]. Similar nonclassical response was found in para-hydrogen clusters [57-59].

However, recent more accurate experiments with ${ }^{4} \mathrm{He}$ have found no measurable period drop that could be attributed to nonclassical rotational inertia. The authors have come to the conclusion that the rotational period drop observed in previous torsional oscillator studies was actually measuring the temperature dependence of structural effects, such as the shear modulus stiffening [60-63], and not superfluid effects. As will be explained in better detail below, the main story appears to rely on dislocations and flux along dislocation cores-effects apparently too small to be visible in rotational experiments.

\section{Shear Modulus Stiffening}

It was experimentally found that below about $200 \mathrm{~K}$ the shear modulus of solid ${ }^{4} \mathrm{He}$ increases with lowering temperature, strongly resembling the temperature behavior of the oscillation frequency. The increase in the shear modulus is likely due to the stiffening of the dislocation network via immobilization of dislocations on defects, such as admixture of ${ }^{3} \mathrm{He}$. The increase can be rather drastic, up to $20 \%$. This increase in the shear modulus mimics the increase of the frequency [64-81]. Thermal conductivity in polycrystalline hexagonal close packed (hcp) ${ }^{4} \mathrm{He}$ also increases [82].

These results agree with the study by Bishop et al. [83] who measured the moment of inertia of hcp ${ }^{4} \mathrm{He}$ crystals from $25 \mathrm{mK}$ to $2 \mathrm{~K}$. With a precision of five parts in $10^{6}$ they found no evidence for a nonclassical rotational inertia. This indicates that if a superfluid solid exists, it has a fraction less than $5 \times 10^{-6}$. Thus, the increase of the oscillator frequency at low temperatures in solid ${ }^{4} \mathrm{He}$ does not signify the arising superfluidity, but is caused by the changes in the shear modulus.

The plastic properties of hcp ${ }^{4} \mathrm{He}$ are different at low temperatures below $0.5 \mathrm{~K}$ and above this temperature [84]. Plasticity involves the motion and multiplication of dislocations. At low temperatures, the solid responds elastically only for strains up to approximately $\epsilon<0.2 \%$. Sudden stress drops emerge at higher strains, accompanied by the acoustic emission expected from dislocation avalanches. The dimension of the slip regions varies over at least 3 orders of magnitude, up to several millimeters. The mobile dislocations travel at speeds close to the sound speed, much faster than the damped dislocations in conventional solids. The low-temperature dislocation avalanches can increase the helium's elastic shear modulus. The avalanches disappear above $0.5 \mathrm{~K}$, replaced by smooth creep deformation involving thermally excited motion of dislocations. A detailed description of experiments on plastic deformation and flow in solid ${ }^{4} \mathrm{He}$ and ${ }^{3} \mathrm{He}$ are described by Beamish [85]. 


\section{Important Role of Disorder}

In the continuing search for possible occurrence of superfluid solids, one usually connects the possible existence of such a matter with the necessity for it to represent in some sense a disordered crystalline structure. It seems that disorder of a crystalline lattice plays a crucial role in the possible appearance of superfluidity in a solid. Theoretical papers claiming that superfluid solids could be perfect crystals [86-91], are usually either based on phenomenological models or use approximate methods allowing for the existence of Bose-Einstein condensate. Among the latter methods, it is possible to mention variational approaches based on Jastrow wave functions or shadow wave functions, which do demonstrate the presence of Bose condensate.

It is now widely accepted that if superfluid effects are expected to arise in a solid, this compulsorily requires the existence of some kind of disorder [92]. For instance, this could be quantum vacancies or interstitials, as has been assumed by Andreev and Lifshitz [35] and others [93-97], dislocations [98], or grain boundaries and surfaces [99-102]. This can be a nanoporous glass, where a local Bose-Einstein condensation in nanopores is observed [103-105], or an amorphous solid with an absolutely irregular structure that is a kind of a glass [106-112].

It is understood that a solid could exhibit superfluid properties only in the presence of some kind of local disorder.

\section{Results of Scattering Experiments}

Solid helium, with both hcp and body-centered cubic (bcc) structures, has been thoroughly investigated by scattering experiments in the range below $200 \mathrm{mK}$ in the attempts to find indications of some changes related to alleged superfluidity in solids. Neutron diffraction experiments, measuring the mean-square atomic displacement in hcp phase solid, found no evidence for an anomaly $[113,114]$.

Neutron scattering experiments, measuring atomic momentum distribution $n(k)$, showed no sign of Bose-Einstein condensation in solid helium [115,116]. Neutron scattering experiments have also been used for studying the dynamic structure factor of amorphous solid helium. No liquid-like phonon-roton modes or other sharply defined modes at low energy or modes unique to a quantum amorphous solid that might suggest superflow are observed [117]. The maximal studied mode energy was lower than $1 \mathrm{meV}$, i.e., $\omega(k)<10 \mathrm{~K}$.

$X$-ray synchrotron radiation was used for measuring peak intensities and lattice parameters of solid ${ }^{4} \mathrm{He}$, showing no indication of superfluid effects [118].

However, there were reports observing, by means of inelastic neutron scattering, structural fluctuations in bcc solid helium $[119,120]$ and coherent delocalized roton-like modes in hcp solid helium associated with delocalized atoms [121]. See also discussion in review [122].

\section{Mass Flux Experiments}

If a solid sample would enjoy superfluid properties, it should exhibit mass transport upon applying a pressure gradient to the sample. This idea was checked in experiments [123] with solid He at low temperature below $200 \mathrm{mK}$, where superfluid properties could be expected. These experiments showed no indication of superfluid flow in response to a pressure difference. The same conclusion of the absence of pressure-driven superfluid flow was obtained in low-frequency experiments [124].

A different setup was used in the experiments of mass flux measurements [125-132] summarized by Hallock [133]. The idea relies on the fact that superfluid helium in a micro-porous environment at a given temperature freezes at an elevated pressure compared with the freezing pressure for bulk ${ }^{4} \mathrm{He}$. The micro-porous material Vycor, filled by superfluid, has a very low thermal conductivity which allows the reservoirs of superfluid at temperatures near $1.5 \mathrm{~K}$ to be available to allow application of pressure and temperature differences between the reservoirs on the two sides of the apparatus, while a much colder region of solid ${ }^{4} \mathrm{He}$ is in between, i.e., it is possible to create an environment in which bulk solid helium, at pressures above the melting curve, can be in direct contact with superfluid 
Helium inside the porous material Vycor. Two Vycor rods containing superfluid Helium were inserted at the ends of a solid sample of Helium filling the horizontal cylindrical region of the cell. The basic question to be answered was whether atoms from one superfluid reservoir would pass into solid helium and as a result, cause atoms to enter the other reservoir.

This setup, called superfluid-solid-superfluid, allows injection of ${ }^{4} \mathrm{He}$ atoms from superfluid directly into the solid hcp ${ }^{4} \mathrm{He}$. Manipulating mass injection and temperature, it is possible to create the chemical potential difference between the sample ends and produce a kind of superfluid flow. Samples grown at lower pressures show flow that depends on sample history, while samples grown at higher pressures show no clear evidence for any such flow. The flow was observed at temperatures below $550 \mathrm{mK}$ and at pressures below approximately 27 bar. Above $600 \mathrm{mK}$, the flux becomes too small to be measured. It falls abruptly in the vicinity of a blocking temperature $T_{d}$ depending on the concentration of the ${ }^{3} \mathrm{He}$ admixture blocking the flux. At low enough concentration the flux rises with lower temperature after the fall, but at high enough concentration of the ${ }^{3} \mathrm{He}$ admixture the flux becomes extinguished.

The absolute value of the measured mass flux, in addition to temperature and applied pressure, depends on the specific sample and its history, but the general temperature dependence is similar for most of the samples studied. A partial anneal of the sample typically causes a reduction in the flux. The magnitude of the flux is measured in units $\mathrm{g}$ per $\mathrm{mm}^{2} \mathrm{~s}$. Thus, in the experiments of Hallock et al. [133] the maximal flux was $1.5 \times 10^{-9} \mathrm{~g} / \mathrm{mm}^{2} \mathrm{~s}$. A much higher flux of approximately $7.2 \times 10^{-8} \mathrm{~g} / \mathrm{mm}^{2} \mathrm{~s}$ was seen for the sample with $8 \mu$ thickness [134]. However, in the latter experiment the mass flow extinction was absent. In the work [135] with solid samples of about $2 \mathrm{~mm}$ thickness, a typical flux of approximately $2.5 \times 10^{-8} \mathrm{~g} / \mathrm{mm}^{2} \mathrm{~s}$ is found. In general, it has been shown that thinner samples result in higher values of the flux, with the mass flow rate decreasing logarithmically with the thickness of the solid ${ }^{4} \mathrm{He}$.

Similar results were obtained in other setups. Thus, the work [136] reports the results of flow experiments in which two chambers containing solid ${ }^{4} \mathrm{He}$ are connected by a superfluid Vycor channel, which is called solid-superfluid-solid junction. In the other experiment [137] the authors observed a flow in the setup where Vycor has been eliminated, allowing study of the intrinsic flow in solid ${ }^{4} \mathrm{He}$ without the complications introduced by the presence of superfluid and the associated solid-liquid interfaces. Applying a pressure gradient at one side, the flow was measured at the other side of the sample. As in previous experiments, the flow appeared below $600 \mathrm{mK}$ and increased with decreasing temperature before it was suppressed at a low temperature $T_{d}$ depending on the concentration of ${ }^{3} \mathrm{He}$ admixture. These measurements show that mass flow in solid helium does not require superfluid leads but can be generated directly by pressure differences created by mechanical compression in bulk solids. The flow that appears below $600 \mathrm{mK}$ is not thermally activated, since its rate increases as the temperature decreases. The flow rate is not proportional to the pressure difference across the solid but rather continues at a high rate and then abruptly stops when the final pressure is reached after a few minutes. This behavior is more typical of superflow than of a viscous liquid or conventional plastic flow.

In the recent work by Shin and Chan [138], it is discovered that blocking at a low-temperature $T_{d}$ of mass flow is a temporary effect. When decreasing temperature, the flow commences below $0.6 \mathrm{~K}$ and increases in magnitude, but then shuts off abruptly below a temperature $T_{d}$ near $0.1 \mathrm{~K}$ depending on the concentration of ${ }^{3} \mathrm{He}$ impurities. The blocking temperature $T_{d}$ is found to increase with the concentration of ${ }^{3} \mathrm{He}$ impurities at the few parts per million level. In the measurements [138] on $2.5 \mathrm{~mm}$ thick solid samples it is found that the mass flow rate reduction and extinction near $0.1 \mathrm{~K}$ happens only when the concentration of the helium gas, $x_{3}$, used to prepare the sample, exceeds respectively $3.5 \times 10^{-4}$ and $2 \times 10^{-3}$. After the extinction, the mass flow shows a gradual but complete recovery with a characteristic time of many hours.

There is growing and substantial evidence that the microscopic origin of the arising flux is due to the superfluid transport along dislocations, as has been suggested by Shevchenko [139-141]. The flux propagates along dislocation cores [142-144]. The extinction of the mass flow is due to the trapping of ${ }^{3} \mathrm{He}$ atoms at the nodes or the intersections of the dislocation network which blocks the transport of 
${ }^{4} \mathrm{He}$ along the network. The slow recovery in the $2.5 \mathrm{~mm}$ samples, observed by Shin and Chan [138], can be due to the migration of the trapped ${ }^{3} \mathrm{He}$ atoms along the dislocation lines and drain into the superfluid inside the porous Vycor glass.

The earlier assumptions that the flux could be due to liquid channels that can form where crystal boundaries meet a container walls have been ruled out by the present experiments. The superfluid fraction, estimated based on the observed mass flux of the order of a few grams per year [125-132], gives the superfluid fraction at the level of $10^{-11}$ to $10^{-10}$, under the assumption that the critical velocity is about $10^{4} \mathrm{~cm} / \mathrm{s}$.

It is important to emphasize that the superflow through the solid in the flow experiments [125-133] has always been observed together with the giant isochoric compressibility regime, or the so-called syringe regime, when atoms of ${ }^{4} \mathrm{He}$ from liquid are injected into the solid. In flow experiments one looks at the system response when pressure or temperature is changed in one reservoir with liquid Helium by monitoring pressure or temperature in the second reservoir. The syringe regime is used for studying the isochoric compressibility, that is, the variation of the density of atoms injected into a constant-volume solid in response to varying the outside chemical potential. This compressibility is found to be comparable to that of liquid ${ }^{4} \mathrm{He}$, even though in an ideal solid it is essentially zero. In the syringe regime crystal growth is possible by the mechanism of dislocation superclimb when edge dislocations add atoms to atomic layers provided there is superfluid atomic transport along their cores [143-147].

The Fermi isotope of ${ }^{3} \mathrm{He}$, in which quantum effects are even more important but superfluidity is not expected, has also been studied [148]. Flow measurements on high purity bcc ${ }^{3} \mathrm{He}$ provide the possibility of a direct comparison to a solid ${ }^{4} \mathrm{He}$, using the same cell in which a superfluid-like response in hcp ${ }^{4} \mathrm{He}$, when pressure differences were applied, was observed. In bcc ${ }^{3} \mathrm{He}$, the mass flow also exists, but with rather different properties. Near melting, the flow is thermally activated, but it decreases monotonically with temperature. The flow rates in the solid are essentially constant below $100 \mathrm{mK}$. The very different behaviors of solid ${ }^{3} \mathrm{He}$ and ${ }^{4} \mathrm{He}$ support the interpretation of superflow in ${ }^{4} \mathrm{He}$. Although superflow is not possible in ${ }^{3} \mathrm{He}$ the temperature-independent flow below $100 \mathrm{mK}$ indicates that the flow in this regime also has a quantum origin, being probably due to the motion of defects and dislocations via thermal activation and tunneling mechanisms. It is possible also to mention the crowdion mechanism [36] when a group of atoms simultaneously slides along a crystallographic axis as one quantum object.

\section{Monte Carlo Simulations}

Solid ${ }^{4} \mathrm{He}$ has been intensively studied by means of Monte Carlo simulations. The fact that a perfect ${ }^{4} \mathrm{He}$ crystal does not support superfluid behavior was confirmed in path integral Monte Carlo simulations [149-151]. For superfluid effects to arise, some kind of disorder is required, e.g., a layered structure or glass-like disorder [152,153]. We recall that throughout the paper we keep in mind self-organized solids, but not systems in external fields preimposing spatial periodicity upon constituents. Systems in external periodic fields certainly can be superfluid [154], which, however, are not real solids.

Vacancies that in first papers were suspected as being able to induce superfluidity, turned out to be attractive producing phase separation instead of forming a superfluid fraction $[155,156]$. However, superfluidity can arise at grain boundaries [157] and in the core of screw dislocations in ${ }^{4} \mathrm{He}[143,158]$. Monte Carlo simulations confirmed that there is no superfluidity in perfect crystals, whether three-dimensional or two-dimensional [159-162]. The most widely accepted point of view is that superfluid flow at low temperatures in ${ }^{4} \mathrm{He}$ occurs along dislocations forming a net $[139-141,145,147]$.

\section{Solids with Regions of Disorder}

As is emphasized above, some kind of disorder is necessary for the occurrence of superfluidity in solids. For example, disorder can be caused by a net of dislocations, with regions of disorder along 
these dislocations. The distribution of the latter in space usually is random. The description of such a solid with randomly distributed regions of disorder consists of two principal problems. One problem is the description of separate dislocations and of causes inducing superfluidity along them, which is discussed above. The other challenge is the statistical theory of a solid sample as a whole, when this sample contains randomly distributed regions of disorder inside it. The second problem, which is considered in the present section, aims at answering the question: how to describe the properties of such a sample on average, when observations are done over the whole sample?

To develop a statistical theory characterizing the sample with random regions of disorder, it is possible to resort to the theory of heterophase systems [163-169]. The main idea of the theory is as follows. If we are interested in average properties of a system with randomly distributed regions exhibiting a phase that is different from the surrounding state, then it is reasonable to average over the random-region locations. Then we obtain a picture of a heterophase system with renormalized characteristics taking into account the existence of two different phases.

One should not confuse the effective heterophase system, resulting from the spatial averaging over mesoscopic regions with different symmetry properties, with a Gibbs mixture of two coexisting macroscopic phases. There are several principal differences between these two cases.

(i) The Gibbs mixture occurs only at phase transitions of first order, while the effective system with nanosize inclusions of another phase can exist in a wide range of thermodynamic parameters. There are numerous examples of such systems. Thus, high temperature superconductors very often, if not always, are the composition of superconducting and normal regions in all range of their existence [170-175]. The other common example is given by ferroelectrics with random regions of paraelectric phase existing in a wide range below the critical temperature, because of which they are called precursors or heterophase fluctuations [176-179]. Several other examples can be found in the review articles [180-182]. Solid ${ }^{4} \mathrm{He}$ with random regions of disorder along dislocations, as in the example considered above, also exists in a wide region of temperature and pressure.

(ii) The Gibbs mixture consists of macroscopic volumes of different phases, while the regions of a competing phase forming heterophase fluctuations inside the system are mesoscopic. These fluctuations correspond to the appearance inside a crystal of regions of local disorder with liquid-like properties. These fluctuations are called mesoscopic since their typical size $l_{f}$ is between the microscopic nearest-neighbor distance $a$ and the macroscopic system size $L$, so that $a \ll l_{f} \ll L$. Often, their lifetime $t_{f}$ also is mesoscopic, being between the microscopic local equilibrium time $t_{l o c}$ and the macroscopic observation time $t_{o b s}, t_{l o c} \ll t_{f} \ll t_{o b s}$. In the case of a solid composed of Bose particles, mesoscopic regions of disorder, stretched along dislocations, can house Bose-Einstein condensate, hence, can become superfluid [167].

(iii) The Gibbs mixture is composed of different phases occupying well defined fixed macroscopic volumes needing no averaging, while the mesoscopic regions of disorder are randomly located in space, which requires averaging over spatial configurations. From the mathematical point of view, the difference between the Gibbs mixture and the averaged effective system is evident. The Hamiltonian of the Gibbs mixture is a linear combination of the phase Hamiltonians, while the renormalized Hamiltonian of the effective averaged system is not a linear combination of the terms related to different phases.

(iv) Coexisting Gibbs macroscopic phases do not influence each other, except a thin layer between them. So that in the thermodynamic limit macroscopic phases are practically independent from each other. However, in a system with randomly distributed mesoscopic regions of disorder, different phases strongly influence each other. The effective renormalized Hamiltonian comes from the procedure of averaging over random locations of disordered regions. As a result of this renormalization the effective Hamiltonian acquires a nonlinear dependence on phase probabilities that are defined by the minimization of the thermodynamic potential of the whole system containing all phases. Therefore, the phase probabilities essentially depend on the properties of all phases. 
Statistical theory of a system, being from one side crystalline, and at the same time containing randomly distributed liquid-like regions, is based on the averaging over the local random configurations [180-182]. This averaging, whose mathematical details can be found in Refs. [167,180], yields the effective Hamiltonian $\widetilde{H}=H_{\text {sol }} \oplus H_{l i q}$, being the direct sum of two replicas, one characterizing crystalline solid state, the other, disordered liquid-like state. The Hamiltonian is defined on the tensor-product space $\widetilde{\mathcal{H}}=\mathcal{H}_{s o l} \otimes \mathcal{H}_{\text {liq }}$, where the first factor is the Hilbert space corresponding to the solid state and the second factor describes the liquid-like state.

The number of particles in the solid phase is

$$
N_{s o l}=\left\langle\hat{N}_{s o l}\right\rangle, \quad \hat{N}_{s o l}=w_{s o l} \int \psi_{s o l}^{\dagger}(\mathbf{r}) \psi_{s o l}(\mathbf{r}) d \mathbf{r},
$$

with $w_{\text {sol }}$ being the probability of the solid state.

In the presence of Bose-Einstein condensate, the liquid-like state must be described in a self-consistent way, preserving the conditions of condensate existence and its stability [183-185]. The field operator of the liquid-like state can be represented be means of the Bogolubov shift $\psi_{\text {liq }}(\mathbf{r})=\eta(\mathbf{r})+\psi_{1}(\mathbf{r})$, with $\eta$ being the condensate function and $\psi_{1}$, the operator of uncondensed particles. The condensate function plays the role of the order parameter describing a system with global gauge symmetry breaking, $\eta(\mathbf{r})=\left\langle\psi_{\text {liq }}(\mathbf{r})\right\rangle$, which implies the statistical average $\left\langle\psi_{1}(\mathbf{r})\right\rangle=0$. The condensate function and the operator of uncondensed particles are mutually orthogonal.

The number of particles in the liquid-like state $N_{l i q}=N_{0}+N_{1}$ is the sum of the number of condensed particles

$$
N_{0}=w_{l i q} \int|\eta(\mathbf{r})|^{2} d \mathbf{r},
$$

and the number of uncondensed particles

$$
N_{1}=\left\langle\hat{N}_{1}\right\rangle, \quad \hat{N}_{1}=w_{l i q} \int \psi_{1}^{\dagger}(\mathbf{r}) \psi_{1}(\mathbf{r}) d \mathbf{r},
$$

where $w_{l i q}$ is the probability of the liquid-like state.

The numbers of particles define the corresponding fractions: the solid-state fraction $n_{s o l} \equiv N_{s o l} / N$, condensate fraction $n_{0} \equiv N_{0} / N$, and the fraction of liquid-like uncondensed particles $n_{1} \equiv N_{1} / N$, where the total number of particles is $N=N_{s o l}+N_{l i q}=N_{s o l}+N_{0}+N_{1}$. The system chemical potential is $\mu=\mu_{0} n_{0}+\mu_{1} n_{1}$. The phase probabilities $w_{\text {sol }}$ and $w_{l i q}$ are found from the minimization of the grand potential of the whole system under the normalization condition $w_{s o l}+w_{l i q}=1$.

There exists a narrow region of the system parameters, where there can arise local Bose-Einstein condensation, hence local superfluidity [167]. If the random superfluid regions form a connected net, there can arise a superfluid flow through the crystalline sample.

\section{Periodic Droplet Structures}

The ground state of a system strongly depends on its parameters and the type of particle interactions. Systems with dipolar interactions have recently become an object of intensive studies [186-192]. Theoretical works hint on the possible existence of periodic ground-state structures with superfluid properties for systems with dipolar interactions [193], as well as for some soft-core potentials [194] and for Rydberg-dressed atoms [195,196]. The phase transition to the periodic state is usually of first order, although there are theoretical speculations on the existence of a critical point, where the transition could become of second order [197].

Theoretical estimates predict a ground-state phase diagram with three distinct regimes: a regular Bose-Einstein condensate, an incoherent array of droplets, and a coherent array of quantum droplets. The coherent droplets are connected by a background condensate, which leads to a phase coherence throughout the whole system. 
Quantum Monte Carlo simulations for dipolar systems with aligned dipolar moments have shown $[198,199]$ that at low temperature there can develop triangular periodic structures composed of filaments or droplets containing many coherent atoms possessing superfluid properties. Between different droplets or across different filaments there can arise quantum-mechanical particle exchanges allowing for the global phase coherence and a superfluid response. This concerns three-dimensional systems, while for two-dimensional dipolar systems there are indications [200] that there is no phase coherence among stripes, hence no global superfluid properties.

Monte Carlo simulations also show that similar effects exist in three-dimensional Bose systems of Rydberg-dressed atoms [201], where there appear periodic droplet structures with phase coherence between droplets. A first-order quantum phase transition from a homogeneous superfluid phase to the periodic droplet phase with face-centered cubic symmetry is predicted.

In experiments, dipolar quantum droplets arranged in periodic arrays have been observed in trapped dipolar Bose-Einstein condensates of ${ }^{162} \mathrm{Dy},{ }^{164} \mathrm{Dy}$ and ${ }^{166} \mathrm{Er}$ [202-205]. The existence of periodic droplet structures of quantum gases with superfluid properties is confirmed by the observation of the low-energy Goldstone mode [204,205].

The dipolar periodic structures are not absolutely stable - their lifetime is limited by fast inelastic losses caused by three-body collisions. Thus, the droplet structure of ${ }^{164}$ Dy survives for $0.1 \mathrm{~s}$ and of ${ }^{166} \mathrm{Er}$, for $0.01 \mathrm{~s}$ [203].

Other conditions for the appearance of droplet periodic structures include the use of a homogeneous external magnetic field controlling the value of the scattering length by means of the Feshbach resonance and polarizing atomic magnetic dipoles in one direction, which is necessary for realizing collective excitations with a roton minimum approaching zero.

Under these conditions (Bose-Einstein condensation, polarization of dipoles, and tuned sufficiently strong dipolar interactions), the atomic system exhibits simultaneous breaking of global gauge symmetry and of spatial uniform symmetry, thus, forming a kind of a superfluid crystal.

\section{Conclusions}

One often describes a superfluid solid as a system, where spatial translational symmetry is spontaneously broken simultaneously with the spontaneous breaking of global gauge symmetry. This definition correctly excludes from the sought objects those where the spatial symmetry is broken not spontaneously but by external fields, like in optical lattices, where the spatial periodicity is superimposed by laser beams.

However, this definition incorrectly excludes nonperiodic solids. Amorphous solids also are solids, not less than periodic crystals. Moreover, as follows from Monte Carlo studies, superfluidity, say in solid helium, is necessarily accompanied by the occurrence of defects disturbing the ideal lattice periodicity. A crystal with defects is only approximately periodic. In that sense, the strict periodicity is not necessary for superfluidity in solids, and even contradictory to it. Vice versa, these studies prove that for the occurrence of superfluidity in solids some breaking of periodicity is required.

Spontaneous breaking of global gauge symmetry is usually the sign for the existence of superfluidity. However, the breaking is not necessary: superfluidity can arise without this symmetry breaking, e.g., in two-dimensional systems. In general, spontaneous breaking of global gauge symmetry is neither necessary nor sufficient for the existence of superfluidity [183].

Mass flux experiments with solid ${ }^{4} \mathrm{He}$ indicate onto the occurrence of superfluid flow through the solid sample. This is a bulk effect, since the mass flow along container walls has been ruled out by the present experiments. There is growing and substantial evidence that mass flow along dislocation cores is responsible for the flux. The occurrence of the flux is accompanied by the so-called syringe effect when the density of a solid can be enhanced by mass injection.

Droplet dipolar crystals serve as another example of periodic systems enjoying superfluid properties. In these crystals, lattice spacing is self-organized, depending on the system parameters. One may say that it is not completely self-organized, since its existence requires a spatial tuning of atomic interactions, low temperature, and polarization of magnetic dipoles. However, actually, 
any crystal requires for its existence some external conditions, such as temperature and pressure. One may say that the droplet dipolar crystal is not absolutely stable, since its lifetime is limited by the decay time caused by three-body collisions. However, this lifetime, although short, anyway, is much longer than the local equilibration time. Therefore, the system can be treated at least as metastable.

Funding: This research received no external funding.

Acknowledgments: The author is grateful to the referees for constructive comments helping to improve the paper. Discussions with E.P. Yukalova are appreciated.

Conflicts of Interest: The authors declare no conflict of interest.

\section{References}

1. Kapitza, P. Viscosity of liquid Helium below the $\lambda$ point. Nature 1938, 141, 74. [CrossRef]

2. Allen, J.F.; Misener, A.D. Flow of liquid Helium II. Nature 1938, 141, 75. [CrossRef]

3. Landau, L. The theory of the superfluidity of Helium II. Phys. Rev. 1941, 60, 356-358. [CrossRef]

4. Landau, L. On the theory of superfluidity Phys. Rev. 1949, 75, 884-885.

5. Landau, L.D. The theory of the superfluidity of Helium II. Zh. Exp. Teor. Fiz. 1941, 11, 592; English translation in Collected Papers of L.D. Landau; Pergamon: Oxford, UK, 1965; pp. 301-330. [CrossRef]

6. Tisza, L. Transport phenomena in Helium II. Nature 1938, 141, 913. [CrossRef]

7. Andronikashvili, E.L. A direct observation of two kinds of motion in helium II. J. Phys. (USSR) 1946, 10, 201-223.

8. Andronikashvili, E.L. Temperature dependence of the normal density of He II. Zh. Exp. Teor. Fiz. 1948, $18,424-431$.

9. Andronikashvili, E.L.; Mamaladze, Y.G. Rotation of Helium II. Prog. Low Temp. Phys. 1967, 5, 79-160.

10. Lifshitz, E.M. Superfluidity. Sci. Am. 1958, 198, 30-35. Reprinted in Perspectives in Theoretical Physics: The Collected Papers of E.M. Lifshitz; Pitaevskii, L.P., Ed.; Pergamon Press Plc: Oxford, UK, 1992; pp. 413-424. doi.org/10.1016/B978-0-08-036364-6.50037-5 [CrossRef]

11. Andronikashvili, E.L. Reflections on Liquid Helium; American Institute of Physics: New York, NY, USA, 1990.

12. Balibar, S. The discovery of superfluidity. J. Low Temp. Phys. 2007, 146, 441-470. [CrossRef]

13. Schmitt, A. Introduction to Superfluidity; Springer: Berlin, Germany, 2014.

14. Prokof'ev, N. What makes a crystal supersolid? Adv. Phys. 2007, 56, 381-402. [CrossRef]

15. Kuklov, A.B.; Prokof'ev, N.V.; Svistunov, B.V. How solid is supersolid? Physics (APS) 2011, 4, 109. doi:10.1103/Physics.4.109. [CrossRef]

16. Boninsegni, M.; Prokof'ev, N.V. Supersolids: What and where are they? Rev. Mod. Phys. 2012, 84, 759-776. [CrossRef]

17. Marchetti, M.C. Frictionless fluids from bacterial teamwork. Nature 2015, 525, 37-39. [CrossRef]

18. Matsuda, H.; Tsumoto, T. Off-diagonal long-range order in solids. Suppl. Prog. Theor. Phys. 1970, 46, 411-436. [CrossRef]

19. Mullin, W.J. Cell model of a Bose-condensed solid. Phys. Rev. Lett. 1971, 26, 611-614. [CrossRef]

20. Oxford Living Dictionaries; Oxford University Press: Oxford, UK, 2018.

21. Bogolubov, N.N. Lectures on Quantum Statistics; Ryadyanska Shkola: Kiev, Ukraine, 1949.

22. Bogoliubov, N.N. Lectures on Quantum Statistics. Volume 1: Quantum Statistics; Gordon and Breach: New York, NY, USA, 1967.

23. Bogoliubov, N.N. Lectures on Quantum Statistics. Volume 2: Quasi-Averages; Gordon and Breach: New York, NY, USA, 1970.

24. Bogolubov, N.N. Quantum Statistical Mechanics; World Scientific: Singapore, 2015.

25. Gross, E.P. Small oscillation theory of the interaction of a particle and scalar field. Phys. Rev. 1955, 100, 1571-1578. [CrossRef]

26. Gross, E.P. Unified theory of interacting bosons. Phys. Rev. 1957, 106, 161-162. [CrossRef]

27. Gross, E.P. Classical theory of boson wave fields. Ann. Phys. (N. Y.) 1958, 4, 57-74. [CrossRef]

28. Gross, E.P. Quantum theory of interacting bosons. Ann. Phys. (N. Y.) 1960, 9, 292-324, [CrossRef]

29. Gross, E.P. Periodic ground states in the many-body problem. Phys. Rev. Lett. 1960, 4, 599-601. [CrossRef]

30. Gross, E.P. Structure of a quantized vortex in boson systems. Nuovo Cimento 1961, 20, 454-477. [CrossRef] 
31. Gross, E.P. Hydrodynamics of a superfluid condensate. J. Math. Phys. 1963, 4, 195-207. [CrossRef]

32. Kirzhnits, D.A.; Nepomnyashchii, Y.A. Coherent crystallization of quantum liquid. J. Exp. Theor. Phys. 1971, 32, 1191-1197. Available online: http:/ / www.jetp.ac.ru/cgi-bin/e/index/e/32/6/p1191?a=list.

33. Vozyakov, V.I. Approximate second quantization method in the quantum crystal theory. Theor. Math. Phys. 1977, 33, 136-144. [CrossRef]

34. Penrose, O.; Onsager, L. Bose-Einstein condensation in liquid helium. Phys. Rev. 1956, 104, 576-584. [CrossRef]

35. Andreev, A.F.; Lifshits, I.M. Quantum theory of defects in crystals. J. Exp. Theor. Phys. 1969, 29, 1107-1113. Available online: http://www.jetp.ac.ru/cgi-bin/e/index/e/29/6/p1107?a=list.

36. Pushkarov, D.I. Quasiparticle Theory of Defects in Solids; World Scientific: Singapore, 1991.

37. Andreev, A.F. Diffusion in quantum crystals. Sov. Phys. Usp. 1976, 19, 137-168. [CrossRef]

38. Andreev, A.F. Quantum crystals. Prog. Low Temp. Phys. 1982, 8, 67-131.

39. Chester, G.V. Topics in the theory of liquid helium four. In Quantum Fluids and Nuclear Matter; Mahanthappa, K.T., Wesley, E.B., Eds.; Gordon and Breach: New York, NY, USA, 1969; pp. 253-296.

40. Chester, G.V. Speculations on Bose-Einstein condensation and quantum crystals. Phys. Rev. A 1970, 2, 256-258. [CrossRef]

41. Meisel, M.W. Supersolid ${ }^{4} \mathrm{He}$ : An overview of past searches and future possibilities. Phys. B 1992, 178, 121-128. [CrossRef]

42. Leggett, A.J. Can a solid be superfluid? Phys. Rev. Lett. 1970, 25, 1543-1546. [CrossRef]

43. Kim, E.; Chan, M.H.W. Probable observation of a supersolid helium phase. Nature 2004, 427, $225-232$. [CrossRef]

44. Kim, E.; Chan, M.H.W. Observation of superflow in solid helium. Science 2004, 305, 1941-1945. [CrossRef]

45. Kim, E.; Chan, M.H.W. Observation of non-classical rotational inertia in solid ${ }^{4} \mathrm{He}$ confined in porous gold. J. Low Temp. Phys. 2005, 138, 859-864. [CrossRef]

46. Kim, E.; Chan, M.H. Supersolid helium at high ptressure. Phys. Rev. Lett. 2006, 97, 115302. [CrossRef] [PubMed]

47. Rittner, A.S.C.; Reppy, J.D. Observation of classical rotation inertia and nonclassical supersolid signals in solid ${ }^{4}$ He below 250 mK. Phys. Rev. Lett. 2006, 97, 165300. [CrossRef]

48. Rittner, A.S.C.; Reppy, J.D. Disorder and the superfluid state of solid ${ }^{4}$ He. Phys. Rev. Lett. 2007, 98, 175302 . [CrossRef]

49. Aoki, Y.; Graves, J.G.; Kojima, H. Oscillation frequency dependence of non-classical rotation inertia of solid ${ }^{4}$ He. Phys. Rev. Lett. 2007, 99, 015301. [CrossRef] [PubMed]

50. Clark, A.C.; West, J.T.; Chan, M.H.W. Nonclassical rotational inertia in helium crystals. Phys. Rev. Lett. 2007, 99, 135302. [CrossRef] [PubMed]

51. Kondo, M.; Takeda, S.; Shibayama, Y.; Shirahama, K. Observation of non-classical rotational inertia in bulk solid ${ }^{4}$ He. J. Low Temp. Phys. 2007, 148, 695-699. [CrossRef]

52. Clark, A.C.; Mynard, J.D.; Chan, M.H.W. Thermal history of solid ${ }^{4}$ He under oscillations. Phys. Rev. B 2008, 77, 184513. [CrossRef]

53. Rittner, A.S.C.; Reppy, J.D. Probing the upper limit of nonclassical rotational inertia in solid helium 4. Phys. Rev. Lett. 2008, 101, 155301. [CrossRef] [PubMed]

54. Mulders, N.; West, J.T.; Chan, M.H.W.; Kodituwakku, C.N.; Burns, C.A.; Lurio, L.B. Torsional oscillator and sychrotron X-ray experiments on solid ${ }^{4} \mathrm{He}$ in aerogel. Phys. Rev. Lett. 2008, 101, 165303. [CrossRef] [PubMed]

55. West, J.T.; Lin, X.; Cheng, Z.G.; Chan, M.H.W. Supersolid behavior in confined geometry. Phys. Rev. Lett. 2009, 102, 185302. [CrossRef] [PubMed]

56. Kitamura, A.; Yagi, M.; Kubota, M.; Shimizu, N. Probable observation of quantized vortex lines trough solid He DC rotation. J. Low Temp. Phys. 2011, 162, 492-499.

57. Clark, A.C.; Liu, X.; Chan, M.H.W. Search for superfluidity in solid hydrogen. Phys. Rev. Lett. 2006, 97, 245301. [CrossRef]

58. Mezzacapo, F.; Boninsegni, M. Local superfluidity of parahydrogen clucters. Phys. Rev. Lett. 2008, 100, 145301. [CrossRef]

59. Li, H.; Le Roy, R.L.; Roy, P.N.; McKellar, A.R.W. Molecular superfluid: nonclassical rotations in doped para-hydrogen clusters. Phys. Rev. Lett. 2010, 105, 133401. [CrossRef] 
60. Kim, D.A.; Chan, M.H. Absence of supersolidity in solid helium in porous Vycor glass. Phys Rev Lett. 2012, 109, 155301. [CrossRef]

61. Kim, D.A.; Chan, M.H.W. Upper limit of supersolidity in solid helium. Phys. Rev. B 2014, 90, 064503. [CrossRef]

62. Choi, J.; Shin, J.; Kim, E. Frequency-dependent study of solid helium-4 contained in a rigid double-torus torsional oscillator. Phys. Rev. B 2015, 92, 144505. [CrossRef]

63. Fear, M.J.; Walmsley, P.M.; Zneev, D.E.; Mäkinen, J.T.; Golov, A.I. No effect of steady rotation on solid ${ }^{4} \mathrm{He}$ in a torsional oscillator. J. Low Temp. Phys. 2016, 183, 106-114. [CrossRef]

64. Day, J.; Beamish, J. Low temperature shear modulus changes in solid ${ }^{4} \mathrm{He}$ and connection to supersolidity. Nature 2007, 450, 853-856. [CrossRef]

65. Corboz, P.; Pollet, L.; Prokof'ev, N.V.; Troyer, M. Binding of a ${ }^{3}$ He impurity to a screw dislocation in solid ${ }^{4}$ He. Phys. Rev. Lett. 2008, 101, 155302. [CrossRef]

66. Bouchaud, J.P.; Biroli, G. Quantum plasticity and dislocation-induced supersolidity. Compt. Rend. Phys. 2008, 9, 1067-1075. [CrossRef]

67. Day, J.; Syshchenko, O.; Beamish, J. Intrinsic and dislocation-induced elastic behavior of solid helium. Phys. Rev. B 2009, 79, 214524. [CrossRef]

68. Day, J.; Syshchenko, O.; Beamish, J. Non-linear elastic response in solid helium: critical velocity or strain? Phys. Rev. Lett. 2010, 104, 075302. [CrossRef]

69. Syshchenko, O.; Day, J.; Beamish, J. Frequency dependence and dissipation in the dynamics of solid helium. Phys. Rev. Lett. 2010, 104, 195301. [CrossRef]

70. Reppy, J.D. Nonsupersolid origin of the nonclassical rotation inertia in a bulk sample of solid ${ }^{4} \mathrm{He}$. Phys. Rev. Lett. 2010, 104, 255301. [CrossRef] [PubMed]

71. Eyal, A.; Pelleg, O.; Embon, L.; Polturak, E. Evidence for a high temperature disorder-induced mobility in solid ${ }^{4}$ He. Phys. Rev. Lett. 2010, 105, 025301. [CrossRef]

72. Su, J.J.; Graf, M.J.; Balatsky, A.V. A glass anomaly in the shear modulus of solid ${ }^{4}$ He. Phys. Rev. Lett. 2010, 105, 045302. [CrossRef] [PubMed]

73. Pratt, E.J.; Hunt, B.; Gadagkar, V.; Yamashita, M.; Graf, M.J.; Balatsky, A.V.; Davis, J.C. Interplay of rotational, relaxational, and shear dynamics in solid ${ }^{4}$ He. Science 2011, 332, 821-824. [CrossRef] [PubMed]

74. Aleinikava, D.; Kuklov, A.B. The role of ${ }^{3} \mathrm{He}$ impurities in the stress induced roughening of superclimbing dislocations in solid ${ }^{4}$ He. J. Phys. Conf. Ser. 2012, 400, 012036. [CrossRef]

75. Mi, X.; Mueller, E.; Reppy, J.D. Study of supersolidity and shear modulus anomaly of ${ }^{4} \mathrm{He}$ in a triple compound oscillator. J. Phys. Conf. Ser. 2012, 400, 012047. [CrossRef]

76. Zhou, C.; Su, J.J.; Graf, M.J.; Reichhardt, C.; Balatsky, A.V.; Beyerlein, I. Dislocation induced anomalous softening of solid helium. Phil. Mag. 2012, 92, 608-616. [CrossRef]

77. Reppy, J.D.; Mi, X.; Justin, A.; Mueller, E.J. Interpreting torsional oscillator measurements: Effect of shear modulus and supersolidity. J. Low Temp. Phys. 2012, 168, 175-193. [CrossRef]

78. Fefferman, A.D.; Rojas, X.; Haziot, A.; Balibar, S.; West, J.T.; Chan, M.H.W. ${ }^{4}$ He crystal quality and rotational response in a transparant torsional oscillator. Phys. Rev. B 2012, 85, 094103. [CrossRef]

79. Eyal, A.; Livue, E.; Polturak, E. Observation of dislocation related interfacial friction mechanism in mobile solid ${ }^{4}$ He. J. Low Temp. Phys. 2016, 183, 31-40. [CrossRef]

80. Tsuiki, T.; Takahashi, D.; Murakawa, S.; Okuda, Y.; Kono, K.; Shirahama, K. Effect of rotation on elastic moduli of solid ${ }^{4}$ He. Phys. Rev. B 2018, 97, 054516. [CrossRef]

81. Choi, J.; Tsuiki, T.; Takahashi, D.; Choi, H.; Kono, K.; Shirahama, K.; Kim, E. Superfluid-like TO responses in rotating solid helium. Phys. Rev. B 2018, 98, 014509. [CrossRef]

82. Zneev, D.B.; Golov, A.I. Simultaneous measurements of the torsional oscillator anomaly and thermal conductivity in solid ${ }^{4}$ He. Phys. Rev. Lett. 2011, 107, 065302. [CrossRef] [PubMed]

83. Bishop, D.J.; Paalanen, M.A.; Reppy, J.D. Search for superfluidity in hcp ${ }^{4}$ He. Phys. Rev. 1981, 24, 2844-2845 [CrossRef]

84. Cheng, Z.G.; Beamish, J. Plastic deformation in a quantum solid: Dislocation avalanches and creep in Helium. Phys. Rev. Lett. 2018, 121, 055301. [CrossRef]

85. Beamish, J. Plastic deformation and creep in solid Helium. J. Low Temp. Phys. 2019, 197, 187-207. [CrossRef]

86. Saslow, W.M. Superflow in solid ${ }^{4}$ He. Phys. Rev. B. 2005, 71, 092502. [CrossRef]

87. Galli, D.E. Bose-Einstein condensation in solid ${ }^{4}$ He. Phys. Rev. B 2005, 71, 140506. [CrossRef] 
88. Saslow, W.M.; Joland, S. Supersolid ${ }^{4}$ He likely has nearly isotropic superflow. Phys. Rev. B 2006, $73,092505$. [CrossRef]

89. Ye, J. Ginzburg-Landau theory of a superfluid. Phys. Rev. Lett. 2006, 97, 125302. [CrossRef]

90. Josserand, C.; Pomeau, Y.; Rica, S. Coexistence of ordinary elasticity and superfluidity in a model of a defect-free supersolid. Phys. Rev. Lett. 2007, 98, 195301. [CrossRef]

91. During, G.; Josserand, C.; Pomeau, Y.; Rica, S. Theory of real supersolids. In Proceedings of Warsaw School of Statistical Physics; Warsaw University: Warsaw, Poland, 2012.

92. Balibar, S.; Caupin, F. Supersolidity and disorder. J. Phys. Cond. Matter 2008, 20, 173201. [CrossRef]

93. Prokof'ev, N.; Svistunov, B. Supersolid state of matter. Phys. Rev. Lett. 2005, 94, 155302. [CrossRef] [PubMed]

94. Galli, D.E.; Reatto, L. Bose-Einstein condensation of incommensurate solid ${ }^{4}$ He. Phys. Rev. Lett 2006, 96, 165301. [CrossRef] [PubMed]

95. Rossi, M.; Vitali, E.; Graf, D.E.; Reatto, L. Zero-point vacancies in quantum solids. J. Low Temp. Phys. 2008, 153, 250-265. [CrossRef]

96. Pessoa, R.; de Koning, M.; Vitiello, S.A. Zero-point vacancy concentration in the shadow wave function model for solid ${ }^{4}$ He. Phys. Rev. B 2009, 80, 172302. [CrossRef]

97. Toda, R.; Gumann, P.; Kosaka, K.; Kanemoto, M.; Onoe, W.; Sasaki, Y. Simultaneous measurement of torsional oscillations and NMR of very dilute ${ }^{3} \mathrm{He}$ in solid ${ }^{4} \mathrm{He}$. Phys. Rev. B 2010, 81, 214515. [CrossRef]

98. Rossi, M.; Vitali, E.; Graf, D.E.; Reatto, L. Quantum dislocations: The fate of multiple vacancies in two dimensional solid ${ }^{4}$ He. J. Phys. Cond. Matt. 2010, 22, 145401. [CrossRef]

99. Dash, J.G.; Wettlaufer, J.S. Classical rotation inertia of solid ${ }^{4}$ He. Phys. Rev. Lett. 2005, 94, 235301. [CrossRef]

100. Penzev, A.; Yasuta, Y.; Kubota, M. ac Vortex-dependent torsional oscillation response and onset temperature $T_{0}$ in solid ${ }^{4}$ He. Phys. Rev. Lett. 2008, 101, 065301. [CrossRef]

101. Gaudio, S.; Cappeluti, E.; Rastelli, G.; Pietronero, L. Finite-size Berezinskii-Kosterlitz-Thouless transition at grain boundaries in solid ${ }^{4} \mathrm{He}$ and the role of ${ }^{3} \mathrm{He}$ impurities. Phys. Rev. Lett. 2008, 101, 075301. [CrossRef] [PubMed]

102. Cappeluti, E.; Rastelli, G.; Gaudio, S.; Pietronero, L. Surface instability and isotopic impurities in quantum solids. Phys. Rev. B 2008, 77, 054301. [CrossRef]

103. Shirahama, K.; Yamamoto, K.; Shibayama, Y. Superfluidity of ${ }^{4}$ He confined in nano-porous media. J. Low Temp. Phys. 2008, 34, 273-278. [CrossRef]

104. Yamamoto, K.; Shibayama, Y.; Shirahama, K. Thermodynamic evidence for nanoscale Bose-Einstein condensation in ${ }^{4} \mathrm{He}$ confined in nanoporous media. Phys. Rev. Lett. 2008, 100, 195301. [CrossRef]

105. Yamamoto, K.; Shibayama, Y.; Shirahama, K. Liquid-solid transition and phase diagram of ${ }^{4} \mathrm{He}$ confined in nanoporous glass. J. Phys. Soc. Jpn. 2008, 77, 013601. [CrossRef]

106. Andreev, A.F. Supersolidity of glasses. JETP Lett. 2007, 85, 585-587. [CrossRef]

107. Grigor'ev, V.N.; Maidanov, V.A.; Rubanskii, V.Y.; Rubets, S.P.; Rudavskii, E.Y.; Ryabalko, A.S.; Syrnikov, E.V.; Tikhii, V.A. Observation of a glassy phase of ${ }^{4} \mathrm{He}$ in the region of supersolid effects. Phys. Rev. B 2007, 76, 224524. [CrossRef]

108. Graf, M.J.; Balatsky, A.V.; Nussinov, Z.; Grigorenko, I.; Trugman, S.A. Torsional oscillators and the entropy dilemma of putative supersolid ${ }^{4}$ He. J. Phys. Conf. Ser. 2009, 150, 032025. [CrossRef]

109. Hunt, B.; Pratt, E.; Gadagkar, V.; Yamashita, M.; Balatsky, A.V.; Davis, J.C. Evidence for superglass state in solid ${ }^{4}$ He. Science 2009, 324, 632-636. [CrossRef]

110. Su, J.J.; Graf, M.J.; Balatsky, A.V. A glassy contribution to the heat capacity of hcp ${ }^{4}$ He solids. J. Low Temp. Phys. 2010, 159, 431-440. [CrossRef]

111. Birshenko, A.P.; Mikhin, N.P.; Rudavskii, E.Y.; Vekhov, Y.O. NMR study of disordered inclusions in the quenched solid helium. J. Low Temp. Phys. 2012, 169, 208-217. [CrossRef]

112. Andreev, A.F. Tunnelling defect nanoclusters in hcp ${ }^{4} \mathrm{He}$ crystals: Alternative to supersolidity. J. Low Temp. Phys. 2012, 168, 126-136. [CrossRef]

113. Balckburn, E.; Goodkind, J.M.; Sinha, S.K.; Hudis, J.; Broholm, C.; van Duin, J.; Frost, C.D.; Kirichek, O.; Down, R.B.E. Absence of low temperature anomaly in the Debye-Waller factor of solid ${ }^{4}$ He. Phys. Rev. B 2007, 76, 024523. [CrossRef]

114. Kirichek, O. Search for supersolid ${ }^{4} \mathrm{He}$ in neutron scattering experiments at ISIS. J. Phys. Conf. Ser. 2009, 150, 032042. [CrossRef]

115. Diallo, S.O.; Pearce, J.V.; Azuah, R.T.; Kirichek, O.; Taylor, J.W.; Glyde, H.R. Bose-Einstein condensation in solid ${ }^{4}$ He. Phys. Rev. Lett. 2007, 98, 205301. [CrossRef] 
116. Diallo, S.O.; Azuah, R.T.; Kirichek, O.; Taylor, J.W.; Glyde, H.R. Limits on Bose-Einstein condensation in confined solid ${ }^{4}$ He. Phys. Rev. B 2009, 80, 060504. [CrossRef]

117. Bossy, J.; Ollivier, J.; Schober, H.; Glyde, H.R. Excitations of amorphous solid helium. Phys. Rev. B 2012, 86, 224503. [CrossRef]

118. Burns, C.A.; Mulders, N.; Lurio, L.; Chan, M.H.W.; Said, A.; Kodituwakku, C.N.; Platzman, P.M. X-ray studies of low-temperature solid ${ }^{4}$ He. Phys. Rev. B 2008, 78, 224305. [CrossRef]

119. Polturak, E.; Markovich, T.; Bossy, J.; Farhi, E. Observation of a new excitation in bcc ${ }^{4} \mathrm{He}$ by inelastic neutron scattering. Phys. B 2003, 329, 371-374. [CrossRef]

120. Pelleg, O.; Shay, M.; Lipson, S.G.; Polturak, E.; Bossy, J.; Marmeggi, J.C.; Kentaro, H.; Farhi, E.; Stunault, A. Observation of macroscopic structural fluctuations in bcc solid ${ }^{4}$ He. Phys. Rev. B 2006, 73, 024301. [CrossRef]

121. Balckburn, E.; Sinha, S.K.; Broholm, C.; Copley, J.R.D.; Erwin, R.W.; Goodkind, J.M. Roton-like mode in solid ${ }^{4}$ He. Pramana J. Phys. 2008, 71, 673-678.

122. Galli, D.E.; Reatto, L. Solid ${ }^{4} \mathrm{He}$ and the superfluid phase: from theoretical description to the discovery of a new state of matter? J. Phys. Soc. Jpn. 2008, 77, 111010. [CrossRef]

123. Day, J.; Beamish, J. Pressure-driven flow of solid helium. Phys. Rev. Lett. 2006, 96, 105304. [CrossRef] [PubMed]

124. Rittner, A.S.C.; Choi, W.; Mueller, E.J.; Reppy, J.D. Absence of pressure driven superfluid flow at low frequency. Phys. Rev. B 2009, 80, 224516. [CrossRef]

125. Ray, M.W.; Hallock, R.B. Observation of unusual mass transport in solid hcp ${ }^{4}$ He. Phys. Rev. Lett. 2008, 100, 235301. [CrossRef] [PubMed]

126. Ray, M.W.; Hallock, R.B. Observation of mass transport through solid ${ }^{4}$ He. Phys. Rev. B 2009, 79, 224302. [CrossRef]

127. Ray, M.W.; Hallock, R.B. Max flux and solid growth in solid ${ }^{4}$ He: 60 mK-700 mK. Phys. Rev. Lett. 2010, 105, 145301. [CrossRef]

128. Vekhov, Y.; Hallock, R.B. Dissipative superfluid mass flux through solid ${ }^{4}$ He. Phys. Rev. B 2014, 90, 134511. [CrossRef]

129. Vekhov, Y.; Mullin, W.J.; Hallock, R.B. Universal temperature dependence, flux extinction, and the role of ${ }^{3} \mathrm{He}$ impurities in superfluid mass transport through solid ${ }^{4} \mathrm{He}$. Phys. Rev. Lett. 2014, 113, 035302. [CrossRef]

130. Vekhov, Y.; Hallock, R.B. Mass superflux in solid helium: the role of ${ }^{3} \mathrm{He}$ impurities. Phys. Rev. B 2015, 92, 104509. [CrossRef]

131. Vekhov, Y.; Hallock, R.B. Flux bottlenecks in the mass superflux in solid helium. Phys. Rev. B 2015, 91, 180506. [CrossRef]

132. Hallock, R.B. Experiments with solid ${ }^{4}$ He. Physica A 2015, 389, 2894-2897. [CrossRef]

133. Hallock, R.B. Mass flux experiments in solid ${ }^{4} \mathrm{He}$ : Some history, recent work and the current status. J. Low Temp. Phys. 2019, 197, 167-186. [CrossRef]

134. Shin, J.; Kim, D.Y.; Haziot, A.; Chan, M.H.W. Superfluidlike mass flow through $8 \mu \mathrm{m}$ thick solid ${ }^{4}$ He samples. Phys. Rev. Lett. 2017, 118, 235301. [CrossRef] [PubMed]

135. Shin, J.; Chan, M.H.W. Mass transport through dislocation network in solid ${ }^{4}$ He. Phys. Rev. B 2019, 99, 140502. [CrossRef]

136. Cheng, Z.G.; Beamish, J.; Fefferman, A.D.; Souris, F.; Balibar, S.; Dauvois, V. Helium mass flow through a solid-superfluid-solid junction. Phys. Rev. Lett. 2015, 114, 165301. [CrossRef]

137. Cheng, Z.G.; Beamish, J. Compression-driven mass flow in bulk solid ${ }^{4}$ He. Phys. Rev. Lett. 2016, $117,025301$. [CrossRef]

138. Shin, J.; Chan, M.H.W. Extinction and recovery of mass flow through solid ${ }^{4}$ He samples. Phys. Rev. B 2020, 101, 014507.

139. Shevchenko, S.I. On one-dimensional superfluidity in Bose crystals. Low Temp. Phys. 1987, 13, $115-131$.

140. Shevchenko, S.I. On quasi-one-dimensional superfluidity in Bose systems. Low Temp. Phys. 1988, 14, 1011-1027.

141. Fil, D.V.; Shevchenko, S.I. Relaxation of superflow in a network: Application to the dislocation model of supersolidity of helium crystals. Phys. Rev. B 2009, 80, 100501. [CrossRef]

142. Pollet, L.; Boninsegni, M.; Kuklov, A.B.; Prokof'ev, N.V.; Svistunov, B.V.; Troyer, M. Local stress and superfluid properties of solid ${ }^{4}$ He. Phys. Rev. Lett. 2008, 101, 097202. [CrossRef] [PubMed]

143. Söyler, S.G.; Kuklov, A.B.; Pollet, L.; Prokof'ev, N.V.; Svustunov, B.V. Underlying mechanism for the giant isochoric compressibility of solid ${ }^{4} \mathrm{He}$ : Superclimb of dislocations. Phys. Rev. Lett. 2009, 103, 175301. [CrossRef] [PubMed] 
144. Kuklov, A.B.; Pollet, L.; Prokof'ev, N.V.; Svustunov, B.V. Quantum plasticity and supersolid response in helium-4. Phys. Rev. B 2014, 90, 184508. [CrossRef]

145. Kuklov, A.B. Plasticity induced superclimb in solid helium-4: Direct abd inverse effects. Phys. Rev. B 2019, 100, 014513. [CrossRef]

146. Kuklov, A.B. Giant isochoric compressibility of solid ${ }^{4} \mathrm{He}$ : The bistability of superclimbing dislocations. Phys. Rev. B 2015, 92, 134504. [CrossRef]

147. Kuklov, A.B.; Prokof'ev, N.V.; Svistunov, B.V. Disorder-induced quantum properties of solid ${ }^{4}$ He. arXiv 2019 , arXiv:1912.03704.

148. Cheng, Z.G.; Beamish, J. Mass flow through solid ${ }^{3}$ He in the bcc phase. Phys. Rev. Lett. 2018, 121, 225304. [CrossRef]

149. Ceperley, D.M.; Bernu, B. Ring exchanges and the supersolid phase of ${ }^{4}$ He. Phys. Rev. Lett. 2004, 93, 155303. [CrossRef]

150. Bernu, B.; Ceperley, D.M. Path integral calculations of exchange in solid ${ }^{4}$ He. J. Phys. Chem. Solids 2005, 66, 1462-1466. [CrossRef]

151. Clark, B.K.; Ceperley, D.M. Off-diagonal long-range order in solid ${ }^{4}$ He. Phys. Rev. Lett. 2006, 96, 105302. [CrossRef] [PubMed]

152. Khairallah, S.A.; Ceperley, D.M. Superfluidity of dense ${ }^{4} \mathrm{He}$ in Vycor. Phys. Rev. Lett. 2005, 95, 185301. [CrossRef] [PubMed]

153. Boninsegni, M.; Prokof'ev, N.; Svistunov, B. Superglass phase of ${ }^{4}$ He. Phys. Rev. Lett. 2006, 96, 105301. [CrossRef]

154. Cazorla, C.; Boronat, J. Superfluidity versus localization in bulk ${ }^{4} \mathrm{He}$ at zero temperature. Phys. Rev. B 2006, 73, 224515. [CrossRef]

155. Boninsegni, M.; Kuklov, A.B.; Pollet, L.; Prokof'ev, N.V.; Svistunov, B.V.; Troyer, M. Fate of vacancy-induced supersolidity in ${ }^{4}$ He. Phy. Rev. Lett. 2006, 97, 080401. [CrossRef]

156. Ma, P.N.; Pollet, L.; Troyer, M.; Zhang, F.C. A classical picture of the role of vacancies and interstitials in helium-4. J. Low Temp. Phys. 2008, 152, 156-163. [CrossRef]

157. Pollet, L.; Boninsegni, A.V.; Kuklov, A.B.; Prokof'ev, N.V.; Svistunov, B.V.; Troyer, M. Superfluidity of grain boundaries in solid ${ }^{4}$ He. Phys. Rev. Lett. 2007, 98, 135301. [CrossRef]

158. Boninsegni, M.; Kuklov, A.B.; Pollet, L.; Prokof'ev, N.V.; Svistunov, B.V.; Troyer, M. Luttinger liquid in the core of screw dislocation in helium-4. Phys. Rev. Lett. 2007, 99, 035301. [CrossRef]

159. Cazorla, C.; Astrakharchik, G.E.; Casulleras, J.; Boronat, J. Ground-state properties and superfluidity of twoand quasi-two-dimensional solid ${ }^{4}$ He. J. Phys. Condens. Matter 2010, 22, 165402. [CrossRef]

160. Boninsegni, M. On the existence of supersolid ${ }^{4}$ He monolayer films. J. Low Temp. Phys. 2011, 165, 67-77. [CrossRef]

161. Rota, R.; Boronat, J. Path integral Monte Carlo calculations of momentum distribution in solid ${ }^{4}$ He. J. Low Temp. Phys. 2011, 162, 146-153. [CrossRef]

162. Rota, R.; Boronat, J. Microscopic approach to the bcc phase of solid ${ }^{4}$ He. Mol. Phys. 2011, 109, $2963-2968$. [CrossRef]

163. Yukalov, V.I. Remarks on quasiaverages. Theor. Math. Phys. 1976, 26, 274-281. [CrossRef]

164. Yukalov, V.I. Model of a hybrid crystal. Theor. Math. Phys. 1976, 28, 652-660. [CrossRef]

165. Yukalov, V.I. Quantum crystal with jumps of particles. Physica A 1977, 89, 363-372. [CrossRef]

166. Yukalov, V.I. A method to consider metastable states. Phys. Lett. A 1981, 81, 433-435. [CrossRef]

167. Yukalov, V.I. Theory of melting and crystallization. Phys. Rev. B 1985, 32, 436-446. [CrossRef]

168. Yukalov, V.I. Properties of solids with pores and cracks. Int. J. Mod. Phys. B 1989, 3, 311-326. [CrossRef]

169. Yukalov, V.I. Possibility of turbulent crystals. Int. J. Mod. Phys. B 2001, 17, 2433-2453. [CrossRef]

170. Phillips, J.C. Physics of High- $T_{c}$ Superconductors; Academic Press: Boston, MA, USA, 1989.

171. Benedek, G.; Müller, K.A. (Eds.) Phase Separation in Cuprate Superconductors; World Scientific: Singapore, 1992.

172. Sigmund, E.; Müller, K.A. Phase Separation in Cuprate Superconductors; Springer: Berlin, Germany, 1994.

173. Kivelson, S.A.; Bindloss, I.P.; Fradkin, E.; Oganesyan, V.; Tranquada, J.M.; Kapitulnik, A.; Howald, C. How to detect fluctuating stripes in the high-temperature superconductors. Rev. Mod. Phys. 2003, 75, 1201-1242 . [CrossRef]

174. Yukalov, V.I.; Yukalova, E.P. Mesoscopic phase separation in anisotropic superconductors. Phys. Rev. B 2004, 70, 224516. [CrossRef]

175. Bianconi, A. Superstripes in the low energy physics of complex quantum matter at the nanoscale. J. Supercond. Nov. Magn. 2015, 28, 1227-1229. [CrossRef]

176. Brookeman, J.; Rigamonti, A. Pretrunsitional clusters and heterophase fluctuations at first-order phase transitions in crystals. Phys. Rev. B 1981, 24, 4925-4930. [CrossRef] 
177. Rigamonti, A. NMR-NQR studies of structural phase transitions. Adv. Phys. 1984, 33, 115-191. [CrossRef]

178. Gordon, A.; Genossar, J. Precursor order clusters at ferroelectric phase transitions. Phys. B 1984, 125, $53-62$. [CrossRef]

179. Gordon, A. Heterophase fluctuations in ferroelectrics. J. Phys. C 1987, 20, 111-114. [CrossRef]

180. Yukalov, V.I. Phase transitions and heterophase fluctuations. Phys. Rep. 1991, 208, 395-489. [CrossRef]

181. Yukalov, V.I. Mesoscopic phase fluctuations: General phenomenon in condensed matter. Int. J. Mod. Phys. B 2003, 17, 2333-2358. [CrossRef]

182. Yukalov, V.I. Systems with symmetry breaking and restoration. Symmetry 2010, 2, 40-68. [CrossRef]

183. Yukalov, V.I. Basics of Bose-Einstein condensation. Phys. Part. Nucl. 2011, 42, 460-513. [CrossRef]

184. Yukalov, V.I. Theory of cold atoms: Basics of quantum statistics. Laser Phys. 2013, 23, 062001. [CrossRef]

185. Yukalov, V.I. Theory of cold atoms: Bose-Einstein statistics. Laser Phys. 2016, 26, 062001. [CrossRef]

186. Griesmaier, A. Generation of a dipolar Bose-Einstein condensate. J. Phys. B 2007, 40, R91. [CrossRef]

187. Baranov, M.A. Theoretical progress in many-body physics with ultracold dipolar gases. Phys. Rep. 2008, 464, 71-111. [CrossRef]

188. Baranov, M.A.; Dalmonte, M.; Pupillo, G.; Zoller, P. Condensed matter theory of dipolar quantum gases. Chem. Rev. 2012, 112, 5012-5061. [CrossRef] [PubMed]

189. Gadway, B.; Yan, B. Strongly interacting ultracold polar molecules. J. Phys. B 2016, 49, 152002. [CrossRef]

190. Stamper-Kurn, D.M.; Ueda, M. Spinor Bose gases: Symmetries, magnetism, and quantum dynamics. Rev. Mod. Phys. 2013, 85, 1191-1244. [CrossRef]

191. Ueda, M. Fundamentals and New Frontiers of Bose-Einstein Condensation; World Scientific: Singapore, 2010.

192. Yukalov, V.I. Dipolar and spinor bosonic systems. Laser Phys. 2018, 28, 053001. [CrossRef]

193. Lu, Z.K.; Li, Y.; Petrov, D.S.; Shlyapnikov, G.V. Stable dilute supersolid of two-dimensional dipolar bosons. Phys. Rev. Lett. 2015, 115, 075303. [CrossRef]

194. Boninsegni, M. Supersolid phases of cold atom assemblies. J. Low Temp. Phys. 2012, 168, 137-149. [CrossRef]

195. Henkel, N.; Nath, R.; Pohl, T. Three-dimensional roton excitations and supersolid formation in Rydberg-excited Bose-Einstein condensates. Phys. Rev. Lett. 2010, 104, 195302. [CrossRef] [PubMed]

196. Cinti, F.; Jain, P.; Boninsegni, M.; Michel, A.; Zoller, P.; Pupillo, G. Supersolid droplet crystal in a dilute-blockaded gas. Phys. Rev. Lett. 2010, 105, 135301. [CrossRef] [PubMed]

197. Zhang, Y.C.; Maucher, F.; Pohl, T. Supersoldity around a critical point in dipolar Bose-Einstein condensates. Phys. Rev. Lett. 2019, 123, 015301. [CrossRef] [PubMed]

198. Cinti, F.; Boninsegni, M. Classical and quantum filaments in the ground state of trapped dipolar Bose gases. Phys. Rev. A 2017, 96, 013627. [CrossRef]

199. Kora, Y.; Boninsegni, M. Patterned supersolids in dipolar Bose systems. J. Low. Temp. Phys. 2019, 197, 337-347. doi:10.1007/s10909-019-02229-z. [CrossRef]

200. Cinti, F.; Boninsegni, M. Absence of superfluidity in 2D dipolar Bose striped crystals. J. Low Temp. Phys. 2019, 196, 413-422. [CrossRef]

201. Seydi, I.; Abedinpour, S.H.; Zillich, R.E.; Asgari, R.; Tanatar, B. Rotons and Bose condensation in Rydberg-dressed Bose gases. Phys. Rev. A 2020, 101, 013628. doi:10.1103/PhysRevA.101.013628. [CrossRef]

202. Bötcher, F.; Schmidt, J.N.; Wenzel, M.; Hertkorn, J.; Guo, M.; Langen, T.; Pfau, T. Transient supersolid properties in an array of dipolar quantum droplets. Phys. Rev. X 2019, 9, 011051.

203. Chomaz, L.; Petter, D.; Ilzhöfer, P.; Natale, G.; Trautmann, A.; Politi, C.; Durastante, G.; Van Bijnen, R.M.; Patscheider, A.; Sohmen, M.; et al. Long-lived and transient supersolid behaviors in dipolar quantum gases. Phys. Rev. X 2019, 9, 021012. [CrossRef]

204. Tanzi, L.; Roccuzzo, S.M.; Lucioni, E.; Fama, F.; Fioretti, A.; Gabbanini, C.; Modugno, G.; Recatti, A.; Stringari, S. Supersolid symmetry breaking from compressional oscillations in a dipolar quantum gas. Nature 2019, 574, 382-385. doi:10.1038/s41586-019-1568-6. [CrossRef]

205. Guo, M.; Böttcher, F.; Herkorn, J.; Schmidt, J.N.; Wenzel, M.; Bichler, H.P.; Langer, T.; Pfau, T. The low-energy Goldstone mode in a trapped dipolar supersolid. Nature 2019, 574, 386-389. doi:10.1038/s41586-019-1569-5. [CrossRef] [PubMed]

(c) 2020 by the author. Licensee MDPI, Basel, Switzerland. This article is an open access article distributed under the terms and conditions of the Creative Commons Attribution (CC BY) license (http:/ / creativecommons.org/licenses/by/4.0/). 